\title{
GESTÃO DO CONHECIMENTO: COMO AS ORGANIZAÇÕES PÚBLICAS DO BRASIL PERCEBEM ESSE MODELO?
}

\section{KNOWLEDGE MANAGEMENT: HOW PUBLIC ORGANIZATIONS OF BRAZIL PERCEIVE THIS MODEL?}

\author{
Ahiram Brunni Cartaxo de Castro ${ }^{1}$; Lydia Maria Pinto Brito ${ }^{2}$ \\ ${ }^{1}$ Instituto Federal de Educação, Ciência e Tecnologia do Rio Grande do Norte - IFRN - Caicó - \\ $\mathrm{RN}$ - Brasil \\ brunnicastro@hotmail.com \\ ${ }^{2}$ Universidade Potiguar - UNP - Natal - RN - Brasil \\ lydiampbrito@yahoo.com.br
}

\begin{abstract}
Resumo
A Gestão do Conhecimento (GC) é o novo modelo de gerir as organizações através da inteligência das pessoas como processo de gerar vantagem competitiva. Nesse sentido questiona-se: como as organizações públicas tem percebido este modelo? O objetivo da pesquisa foi levantar como as organizações públicas brasileiras percebem o modelo da GC. A metodologia utilizada foi estudo exploratório por meio de uma pesquisa bibliográfica em trabalhos disponíveis em meio impresso e digital nos portais acadêmicos na internet sobre GC em organizações públicas no brasil. Os resultados demonstram que: não há na grande maioria das organizações pesquisadas nem explicitação nem formalização da GC. Além disso, com relação à intenção estratégica das práticas, a GC ainda não é uma prioridade para a maioria das organizações públicas, que ainda focam suas ações nos processos táticos para resolução dos problemas do dia a dia, em atividades de obtenção, facilitação, aprendizagem, armazenamento e disseminação do conhecimento.
\end{abstract}

Palavras-chave: gestão do conhecimento; estudo exploratório; organizações públicas brasileiras.

\section{Introdução}

O contexto histórico demonstra que, as práticas ou estratégias de gestão, tais como: reestruturações, reengenharias, fusões, gestão por processos etc., são decorrentes de mudanças macroambientais (que ocorrem por fatores legais/políticos, internacionais, tecnológicos, fatores econômicos, sociais e no ambiente natural - que afetam todas as organizações), e tornaram obsoletas as práticas gerenciais anteriores, ocorrendo-se a quebra de paradigma à medida da evolução dos novos modelos de se administrar as organizações. (TRIGUEIRO; MARQUES, 2012).

A atual demanda - a Gestão do Conhecimento (GC), emergiu a partir dos anos 90 (Era da Competitividade) com o processo da globalização, momento que o conhecimento atingiu maiores 
proporções de desenvolvimento, motivado pela informática, microeletrônica e biotecnologia, principalmente a internet, fomentando-se a disseminação da informação e do conhecimento de forma ilimitada e descentralizada, gerando-se a inovação para as organizações através do capital intelectual dos indivíduos.

A GC, portanto, é um novo modelo de gerir as organizações através da inteligência das pessoas, diga-se dos trabalhadores já que, as organizações devem estar focadas na utilização da informação pelo indivíduo como processo de gerar vantagem competitiva.

Sendo a GC o modelo contemporâneo de gerir as organizações, a pergunta que aflora é: como as organizações públicas tem percebido este modelo?

Assim, este artigo tem como objetivo, a partir de uma revisão bibliográfica sobre GC em organizações públicas no Brasil, responder a esta pergunta. Com esta intenção, parte-se do arcabouço teórico de base interdisciplinar da GC.

Trata-se de um estudo exploratório por meio de uma pesquisa bibliográfica que, segundo Gil (2008, p. 50), "é desenvolvida a partir de material já elaborado, constituído de livros e artigos científicos".

Foram utilizados 44 referências, entre livros, artigos e dissertações de mestrado, publicados entre 1973 e 2015 e contempla trabalhos de autores clássicos e contemporâneos das áreas de administração, ciência da informação, sociologia e educação, disponíveis em meio impresso e digital nos portais acadêmicos na internet.

Os dados foram coletados através de leitura exploratória, leitura seletiva e registradas através de fichamentos para posterior extração.

\section{Movimento dos novos modelos em direção da Gestão do Conhecimento}

O movimento dos modelos de gestão para a GC, tem quatro fases básicas, sendo: $1^{\text {a }}$ fase Definição da competência essencial; $2^{\text {a }}$ fase - Definição do perfil de competência profissional; $3^{\text {a }}$ fase - Definição do perfil de competência gerencial e $4^{\mathrm{a}}$ fase - Da gestão por competências à gestão do conhecimento. Portanto, de forma breve, tem-se:

- $1^{\text {a }}$ fase - Definição da competência essencial: se inicia com a discussão sobre competências, que surgiu no início dos anos 90, com a definição da competência essencial para as organizações colocada por Hamel e Prahalad (1995). Segundo os autores, competência essencial é o conjunto de competências centrais (e não periféricas) compostas de: habilidades pessoais, de habilidades de promoção de uma unidade organizacional e de tecnologias de uma empresa, que representam a soma do aprendizado construído ao longo de sua história na realização de negócios no mercado, e que faz com que seus produtos sejam percebidos e reconhecidos pelos clientes e concorrentes como vantagem significativa 
e como produtos agregadores, em termos de qualidade e custos, de benefícios fundamentais e de valores, produtos com diferenciação qualitativa e que não pode ser facilmente copiada entre os concorrentes.

No Brasil, esse termo - competência essencial foi introduzido por Fleury (1995), como identidade institucional, como sendo a atividade de identificar a principal habilidade da empresa, propor a estratégia de crescimento por segmento de atuação e estabelecer o objetivo financeiro no longo prazo.

Segundo Oliveira (2004) a missão deve ser descrita em termos simples: qual o propósito (competência essencial ou identidade institucional) da empresa? Nesse sentido, quando as organizações definiam seus focos de atuação, passariam a iniciar o processo de mudança na lógica de funcionamento: de especialista-funcionalista dentro da tradição taylorista-fordista para processuais, virtuais e em rede, adotando modelos flexíveis de gestão e "mais adequados" as novas tendências do mercado mundial, complexo e demasiadamente competitivo.

- $2^{\mathrm{a}}$ fase - Definição do perfil de competência profissional: se consolidou na década seguinte (nos anos 2000), e seu objetivo era definir o perfil de competências profissional e os amplos espaços organizacionais profissionais dos trabalhadores.

Na segunda fase, os atributos da antiga lógica, tais como: foco no produto, especialização, hierarquia rígida, departamentalização, chefias bem definidas e equipes especializadas, vão sendo substituídos pelo foco no mercado, pelos times de aprendizagem ${ }^{1}$ com liderança compartilhada, estruturas enxutas, processuais, em rede, virtuais e comportamentos, diga-se habilidades, de saber aplicar conhecimentos e atitudes, que se referem ao querer fazer e o saber ser, que viabilizam os resultados das organizações.

Nesse momento, várias discussões surgem. Nos Estados Unidos o foco do debate, levantado por McClelland (1973) e Boyatizis (1982), estava na orientação para as necessidades do mercado e no relacionamento do desenvolvimento de competências no trabalho com a remuneração estratégica, variável e por adquirir estratégias. Na Europa, a discussão foi levantada principalmente pelos autores Zarifian (2001), Perrenoud (2002) e Le Boterf (2003), acerca da relação entre o desenvolvimento integral do indivíduo ao longo de sua vida versus as políticas governamentais e educacionais para os trabalhadores. No Brasil, surgem duas discussões, sendo: uma levantada por Fleury (1995) e Dutra (2002; 2004), que incorporavam elementos das matrizes americanas e

\footnotetext{
${ }^{1}$ Pessoas com competências diferentes que se unem temporariamente em torno de uma atividade para cumpri-la, ou seja, equipes com multi-habilidades multiprofissionais em instituições flexíveis, horizontalizadas, que através do trabalho cooperativo e complementar se juntam para cumprir uma missão/tarefa.
} 
europeias - que eram consideradas as discussões mais aceitas pelas faculdades de Administração; e, as das faculdades de Educação, que tinham uma orientação mais marxista, preocupadas com a alienação do trabalhador e com a empregabilidade em decorrência da aquisição de competências, levantadas por Frigoto et al. (1995); Frigoto (1999), Ramos (2001) e Brito (2005).

- $\quad 3^{\mathrm{a}}$ fase - Definição do perfil de competência gerencial. Em complemento da $2^{\mathrm{a}}$ fase, esta fase tinha como objetivo desenvolver os conhecimentos, habilidades e atitudes gerenciais, ou seja, preocupava-se com a formação de líderes comprometidos com o processo de mudança instalado naquele momento e capazes de desenvolver a competências dos empregados e obter o comprometimento com a busca de soluções para os problemas empresariais emergentes e críticos e, consequentemente, viabilizar a competência essencial das organizações. (QUINN, 2004; RUAS et al., 2005).

Portanto, entende-se que as fases citadas aqui, compõem um conjunto de matrizes que estão gerando o novo modelo de gestão do trabalho em construção na atualidade em substituição aos modelos burocráticos, ou seja, faz parte de um grande movimento que tem dado fundamento à GC.

- $4^{\text {a }}$ fase - Da gestão por competências à gestão do conhecimento. Trata-se da conexão entre a gestão por competências com a GC, ou seja, a organização define sua identidade/ competência essencial para seu planejamento estratégico (missão, visão, intenções, objetivos etc.), em seguida elabora o perfil de competência profissional dos seus trabalhadores para cumprimento de suas estratégias, gerencia o conhecimento contido nos trabalhadores através dos seus gestores para incorporá-lo nos produtos, serviços e processo de tomada de decisão, e o próximo passo, passa a ser o gerenciamento do conhecimento - tácito e explícito, que trataremos mais tarde, combinado com a tentativa de promover mudança na cultura organizacional, com a intenção de construir uma lógica capaz de unir os trabalhadores em torno da execução dos objetivos organizacionais, em detrimento dos objetivos individuais dos trabalhadores.

Portanto, esse conjunto de fases formam um grande movimento cujo foco é o gerenciamento do conhecimento - mapear, captar, disseminar, reter, construir, avaliar, recompensar e descartar, e as organizações de aprendizagem, que tratam da mudança na cultura organizacional, levantado por Senge (1995), de especialista funcionalista para flexível, como já frisado anteriormente. (grifo nosso). 
Assim, as organizações estão sendo compelidas a cuidar do conhecimento que existe na mente dos seus trabalhadores, como um novo tipo de ativo empresarial. Dessa forma, a GC é o modelo de um processo articulado e intencional, destinado a sustentar ou promover o desempenho global das organizações pelo conhecimento, fazendo as organizações agirem de forma mais inteligente pela facilitação da criação, acumulação, desenvolvimento e uso do conhecimento no ambiente organizacional.

A GC organizacional se ocupa dos processos gerenciais e infra-estrutura física e digital que facilitam, favorecem e estimulam os processos humanos de criação, compartilhamento e uso de conhecimentos individuais e coletivos. (TERRA, 2005). Tem haver com um esforço para usar e manter de forma sistemática, as inovações geradas na empresa para um melhor posicionamento de mercado. (TAVARES, 2010).

Para Drucker (2000); Choo (2003); Brito (2005); Melhor (2010); Brito, Oliveira e Castro; Marques (2015), a GC exige integração, multidisciplinariedade e um processo sistemático de descobrir, criar continuamente novos conhecimentos, mapear na sociedade, classificar, trazer para a organização, disseminar, multiplicar, recompensar e reter o conhecimento incorporando-o velozmente em novos produtos/serviços, tecnologias e sistemas - perpetua a mudança no interior da organização. No entanto, Brito (2005); Melhor (2010); Marques (2015), ressaltam a importância desse conhecimento capturado, ser necessário ou está alinhado à cultura e aos objetivos estratégicos da empresa.

\section{Gestão do Conhecimento em organizações públicas brasileiras}

No Brasil, um dos primeiros movimentos em direção a GC, conforme Cavalcanti et al. (2002), iniciou-se em 1998, mas somente se consolidou a partir dos anos 2002, que foi o curso de pós-graduação lato sensu em Gestão do Conhecimento e Inteligência Empresarial criado pela COPPE da Universidade Federal do Rio de Janeiro.

A COPPE tinha ações voltadas para a sensibilização das pessoas e organizações sobre a importância da GC para o sucesso das organizações no século XXI e apesar de vários profissionais formados em diversas turmas com a consolidação da citada pós-graduação; os estudos, normalmente tinham como foco as organizações de fora do Brasil e privadas.

Em paralelo, conforme Schlesinger et al. (2008), existia um projeto nacional, coordenado pelo Instituto Brasileiro de Informação em Ciência e Tecnologia (IBICT), que originou o Programa "Sociedade da Informação", lançado pelo Governo Federal no ano 2000. Esse programa possui foco em tecnologia da informação para disseminação e armazenamento do conhecimento através do egov (Programa de Governo Eletrônico Brasileiro). 
Porém, somente em 2002, conforme Cavalcanti et al. (2002), foi produzido o primeiro estudo expressivo em uma organização pública no Brasil, sendo o caso da Empresa RIOLUZ do Rio de Janeiro, organização do ramo de energia.

Em paralelo, também conduzido pela COOPE/UFRN, no mesmo ano, foi desenvolvido um estudo, menos expressivo, na EMAE - Empresa Metropolitana de Águas e Energia S.A., empresa de economia mista, controlada pelo Governo de São Paulo do ramo de energia.

Ambos os estudos tinham foco nos processos de identificar os gaps de gestão do capital intelectual, de treinamento dos trabalhadores e gestão documental associado ao serviço que prestavam.

Ainda em 2002, segundo Batista (2012), foi também estudado o Hospital de Clínicas de Porto Alegre (HCPA), uma organização do ramo de saúde, quanto à metodologia que era utilizada pelo hospital para o desenvolvimento, implementação e avaliação de prontuários assistenciais (PAs). A experiência do HCPA com a definição de objetivos terapêuticos e uma sequencia temporal de cuidados e estratégias diagnósticas e terapêuticas, a partir dos PAs, tinha como resultados esperados, segundo Polanczyk et al. (2002) o melhoramento clínico dos pacientes e permitir que mais pacientes se beneficiassem do conhecimento científico sobre as melhores práticas disponíveis.

Portanto, o estudo permitiu identificar os principais processos de GC, sendo: identificação, criação, armazenamento, compartilhamento e aplicação do conhecimento; permitindo-se a criação de novos conhecimentos, ideias, estabelecer novas maneiras de executar os processos de trabalho e introduzir novas práticas de gestão. (BATISTA, 2012).

O estudo ainda permitiu, conforme Batista (2012), a externalização do conhecimento tático que deixou de ser individual e permitiu o compartilhamento do conhecimento dos profissionais mais experientes do corpo clínico do hospital para os menos experientes.

O estudo desenvolvido no HCPA, portanto, além de beneficiar os pacientes do hospital, também beneficiou os servidores, gestores, equipes de trabalho, a organização e a sociedade de uma maneira geral, em virtude da ampliação das competências (conhecimentos e habilidades).

Essa intercessão, ou seja, a soma do conhecimento dos indivíduos, equipes e organização que resultou no aumento da capacidade da sociedade de tratar as patologias comtempladas nos PAs, é o que Batista (2012) chamou de capacidade social. (grifo nosso)

Em 2003, o destaque foi para os estudos de Bose (2003), aplicados ao Instituto Nacional do Câncer (Inca), órgão auxiliar do Ministério da Saúde no desenvolvimento e coordenação das ações integradas para a prevenção e o controle do câncer no Brasil.

O estudo de Bose (2003), inovou, na época, ao propor o caminho da implementação de um modelo de sistema de GC com quatro passos, sendo: criação, estruturação, compartilhamento e 
aplicação do conhecimento, elaborado a partir das contribuições de Thomas Davenport e Laurence Prusak, que descreveram os quatro maiores objetivos dos sistemas de GC para organizações privadas, como sendo: criar a substituição do conhecimento, aumentar o acesso ao conhecimento, melhorar o ambiente do conhecimento e gerenciar o conhecimento como um ativo intangível.

Outro órgão pioneiro na implantação e pesquisa em GC na Administração Pública foi o Ipea - Instituto de Pesquisa Econômica Aplicada, que mantinha um Comitê Técnico de Gestão do Conhecimento e Informação Estratégica em 2004, tinha o objetivo propor normas, recomendações e diretrizes para a política de governo eletrônico em Gestão do Conhecimento. Além disso, buscavam sistematicamente identificar, acompanhar e compartilhar as melhores práticas de GC entre os atores do Governo Eletrônico. (BATISTA, 2004).

Um dos estudos pioneiros sobre o tema foi conduzido por Batista (2004), pesquisador do Ipea, com o objetivo de discutir a importância dessa epistemologia para a Administração Pública e identificar o estágio de sua implementação em 6 organizações da Administração Indireta, que haviam declarado ter implantado práticas de GC espelhadas nas experiências do setor privado, sendo: Banco do Brasil (BB), Caixa Econômica Federal (Caixa), Serviço Federal de Processamento de Dados (SERPRO), Petróleo Brasileiro S.A. (Petrobras), Empresa Brasileira de Pesquisa Agropecuária (Embrapa) e Banco Central do Brasil (Bacen).

Na ocasião, a publicação produzida por Batista (2004, p.8), destacou:

(...) algumas práticas de sucesso e situou o tema da CG nas organizações públicas no campo de estudo da Gestão Pública, defendendo a tese de que a finalidade da Gestão do Conhecimento em tais organizações deve ser vista de forma mais ampla que em empresas do setor privado.

Em paralelo, Gomes (2005), havia desenvolvido um estudo no Poder Judiciário do Estado do Rio de Janeiro (PJERJ) com o objetivo de fomentar a discussão sobre o tema GC e sua importância para o Judiciário Fluminense. Tal estudo fora motivado pela criação inovadora, à época, do Cargo de Diretor Geral de GC.

Em 2005, conforme Batista et al. (2005), também conduzido pelo Ipea, juntamente com a Pontifícia Universidade Católica do Paraná (PUC/PR) e com a Empresa TerraForum Consultores, foi desenvolvido um novo estudo, dessa vez, com 28 órgãos da Administração Direta e em 6 empresas estatais do Executivo Federal Brasileiro, entre elas: Casa Civil da Presidência da República (CC/PR), Comando da Aeronáutica (Comaer), Ministério do Planejamento, Orçamento e Gestão (MP), Banco do Brasil (BB), Empresa Brasileira de Correiros e Telégrafos (ECT) etc.

O segundo estudo, tinha como principais objetivos analisar a situação das práticas de GC na Administração Pública Direta do Governo Federal, comparar as estratégias de implementação da GC e apresentar recomendações à Secretaria de Gestão do Ministério do Planejamento, Orçamento 
e Gestão (Seges/MP) para elaboração e implementação de uma política de Gestão do Conhecimento para o setor público. (BATISTA et al., 2005).

A partir dos estudos desenvolvidos pelo Ipea e seus parceiros, de acordo com Batista (2004); Batista et al. (2005); Schlesinger et al. (2008); Strauhs et al. (2012), percebeu-se que algumas organizações públicas estavam utilizando-se de estratégias para melhor aproveitar seu capital humano, por exemplo: através de equipes multidisciplinares; investimento em comunidades de prática, normalmente iniciados por funcionários que já se comunicam entre si porque compartilham as mesmas práticas, interesses ou objetivos; o compartilhamento do conhecimento com as "best practices" e recompensas à colaboração dos funcionários com reconhecimento público, além de, aumentarem oportunidades para o autodesenvolvimento e treinamento.

Um terceiro estudo-base, conduzido por Batista (2006) juntamente com o Ipea e parceiros, visava analisar como as áreas de administração e de planejamento de Instituições Federais de Ensino Superior (IFES) tratavam o tema GC. Além disso, se propunha a avaliar a situação atual da implantação de práticas de GC nessas organizações; comparar se a situação da implantação de iniciativas de GC nas Ifes com o estágio em que se encontra a adoção de tais práticas nos órgãos e nas entidades da Administração Direta Federal; e, avaliar o grau em que as instituições se encontravam em relação à formalização e à explicitação da GC.

Participaram do referido estudo 45 IFES, sendo: Centro Federal de Educação Tecnológica de Minas Gerais (Cefet/MG), Fundação Universidade Federal de Rio Grande (Furg), Universidade de Brasília (UnB), Universidade Federal da Bahia (UFBA) Universidade Federal de Sergipe (UFS), só para citar.

Os resultados da pesquisa demonstraram que a implantação de práticas de GC nas áreas de administração e de planejamento das Ifes se encontrava no estágio inicial nas três categorias analisadas no estudo, e os melhores eram de ações que estavam planejadas e/ou em implantação, conforme abaixo. Portanto, ainda não eram ações consolidadas.

A comparação dos resultados desta pesquisa com os dos estudos anteriores, publicados pelo Instituto de Pesquisa Econômica Aplicada (Ipea), mostram que tais áreas apresentam índices de implantação inferiores aos dos órgãos e entidades da Administração Direta Federal, por exemplo, na maioria das práticas de GC, apesar de se tratarem de instituições de ensino, cujo principal recursos é o conhecimento.

Com relação à intenção estratégica, assim como os resultados encontrados nos demais estudos conduzidos junto ao Ipea, a GC não é era uma prioridade para a maioria das áreas administrativa e de planejamento das Ifes. Detectou-se também que não há, na grande maioria das áreas de administração e de planejamento das Ifes, nem explicitação nem formalização da gestão do conhecimento. (BATISTA, 2006). 
Um quarto estudo também publicado Batista et al. (2007) e lançado junto ao Ipea e parceiros, buscou avaliar como o tema GC era tratado em organizações públicas de saúde (Centros de pesquisa clínica - unidades vinculadas a hospitais universitários e a outras organizações de saúde) e organizações adesas ao Programa Nacional de Gestão Pública e Desburocratização GesPública).

Participaram da pesquisa 24 organizações públicas do ramo de saúde, sendo: Hospital das Clínicas da Universidade Federal de Minas Gerais (HC/UFMG), Hospital das Clínicas da Faculdade de Medicina de Ribeirão Preto da Universidade de São Paulo (HC-FMRP/USP), (HUCFF/UFRJ), Instituto Fernando Figueiras da Fundação Oswaldo Cruz do Ministério da Saúde, Laboratório Químico Farmacêutico do Exército (LQFE/RJ), entre outros.

Os resultados encontrados por Batista et al. (2007), demonstraram a mesma tendência dos estudos anteriores, pois a maior parte dos centros de pesquisa clínica e das organizações adesas ao GesPública, estão em um estágio inicial do processo de institucionalização da GC, e os melhores resultados eram de ações que estavam planejadas e/ou em implantação, conforme abaixo. Portanto, ainda não se constituíam ações consolidadas.

A partir do ano de 2008, se destacam os estudos conduzidos por Angeloni et al. (2008) que catalogaram 15 casos em GC em empresas públicas, entre elas: SERPRO, CAIXA, CHESF, EMBRAPA etc., e os resultados seguiram a mesma tendência dos estudos anteriores quanto as práticas empreendidas pelas organizações estudadas.

Outro estudo de destaque, foi o conduzido por Runte (2011), em um período em que já havia um grande desenvolvimento conceitual da GC no serviço público no pais. O estudo tinha por objetivo analisar e identificar os desafios para a construção de um modelo integrado de GC para a Agência Nacional de Saúde Suplementar (ANS).

Enquanto inicialmente o objetivo dos estudos sobre GC nas organizações era de propor e discutir a importância dessa epistemologia, de fomentar a discussão sobre o tema, criar condições para propiciar a captação do conhecimento, desenvolver algumas práticas e analisar a situação das práticas de gestão do conhecimento. O estudo de Runte (2011) demonstrou o avanço da discussão sobre o tema no Brasil, pois já trouxe como objetivo do estudo modelos de GC adequados as organizações públicas.

Porém, os resultados do estudo apontaram quase que instantaneamente para a crise de eficácia e legitimidade do setor público frente a seus beneficiários, pois ficou demonstrado conforme Runte (2011) que, a existência de uma cultura organizacional ainda fragmentada é um fator agravante no estabelecimento de um ambiente favorável à implantação de um modelo integrado de GC. Alguns gestores reconhecem que para a ANS ter uma verdadeira GC precisa ter, primeiro, uma visão, uma cultura de unidade institucional. 
Além disso, os mecanismos utilizados para gerir o conhecimento institucional se situam em áreas distintas, não são difundidos para a instituição, são parciais no sentido de não estarem conectados com as demais dimensões da GC. Na ANS, há que se discutir a própria identidade dos Sistemas de Informação, pelas expectativas das diretorias e das gerências-gerais com relação ao que elas esperam de serviços ou da prestação de serviços de tecnologia.

Outro desafio identificado por Runte (2011), diz respeito a existir incentivos à participação de servidores no compartilhamento de informações e nas discussões para elaboração de entendimentos unificados em determinado assunto.

Schlesinger et al. (2008), ainda acrescenta a Prefeitura de Curitiba, como exemplo de organização pública que tem instigado práticas de GC, porém seus resultados segue a tendência dos demais estudos.

Portanto, os estudos realizados pelos autores citados anteriormente, destacaram que as principais práticas em GC por organizações públicas quanto a trabalhadores do conhecimento, são: equipes multidisciplinares, melhores práticas, fóruns, educação corporativa, universidade corporativas, em termos de práticas relacionadas à gestão de RH. Quanto a práticas relacionadas a processos facilitadores da GC, destacam-se: benchmarking, mapeamento do conhecimento, comunidades de práticas, enterprise resource planning, gestão por competências, comunidades por grupos focais e virtuais etc.

Entre as práticas mais utilizadas que se relacionam com a base tecnológica e funcional de suporte à Gestão do Conhecimento, tem-se: Portais /intranets /extranets, gestão eletrônica de conteúdos e documentos, Sistemas de workflow e data warehouse.

Apesar dos resultados em estudos encontrados, de acordo com Batista et al. (2007), de forma generalizada, as práticas de gestão identificadas, se encontram ainda nos estágios iniciais de desenvolvimento e implementação, e existem lacunas significativas na aplicação da maioria delas, inclusive relacionadas com a cultura das organizações. Essa conclusão também se aplica a outros estudos que seguiram a mesma tendência. (COSTA et al., 2009; CASTRO, 2011; OLIVEIRA, 2011; GALVÃO, 2012; GONZAGA, 2014; COSTA, 2014; SANTOS, 2014).

Com relação à intenção estratégica das práticas, a GC ainda não é uma prioridade para a maioria das organizações públicas, que ainda focam suas ações de GC nos processos táticos, de obtenção do conhecimento para resolução dos problemas do dia a dia, em atividades de obtenção, facilitação, aprendizagem, armazenamento de conhecimento e disseminação. Detectou-se também que não há na grande maioria das organizações pesquisadas, nem explicitação nem formalização da gestão do conhecimento. (BATISTA, 2006).

Apesar disso, são organizações públicas que perceberam que poderiam, de alguma forma, facilitar e acelerar o aprendizado organizacional, transformando o conhecimento individual de seus 
trabalhadores em conhecimento coletivo da organização, trazendo, dessa forma, inovações aos produtos, serviços e processos da organização.

\section{Considerações finais}

Portanto, conforme os estudos desenvolvidos por vários autores ao longo do tempo demonstraram que há muito por fazer para implantar com sucesso a GC na em organizações públicas da Administração Indireta; da Administração Direta e em empresas estatais; nas áreas de administração e de planejamento de Instituições Federais de Ensino Superior (IFES); na maior parte das organizações públicas de saúde (Centros de pesquisa clínica - unidades vinculadas a hospitais universitários e a outras organizações de saúde), em organizações adesas ao Programa Nacional de Gestão Pública e Desburocratização - GesPública), em empresas públicas, e em agências nacionais de regulação públicas, pois as organizações públicas pesquisadas parecem não estar totalmente motivadas a buscar o cenário da GC para lidar com a pressão social.

Revela-se então, a partir dos resultados encontrados na pesquisa que, apesar do cenário que consta na literatura - GC e na prática de muitas organizações, nas organizações estudadas a esse modelo ainda não é totalmente determinante na capacidade delas e seus servidores/trabalhadores lidarem com o ambiente que se modifica e se transforma aceleradamente e com a crescente complexidade.

Portanto, tem-se que o desafio das organizações públicas brasileiras ainda está em superar a letargia conceitual sobre o tema GC, as limitações de se enxergar a visão do conhecimento na gestão pública, construir uma cultura organizacional favorável à avaliação do conhecimento, fornecer condições facilitadoras para a avaliação do conhecimento, alinhar a captação de novos conhecimentos necessários para manter as organizações no futuro com o planejamento estratégico, iniciar um esforço educacional com os trabalhadores, criar novos cargos de gestor do conhecimento, reconhecer as pessoas fiéis e com conhecimento de valor agregado para as organizações etc., como forma de viabilizar a diferenciação através da inovação, tratar da formalização da GC nas organizações e se utilizar do novo modelo de forma estratégica.

\footnotetext{
Abstract

The Knowledge Management (KM) is the new model of managing organizations through the intelligence of the people as a process of generating competitive advantage. In this sense it questions: how public organizations have realized this model? The objective of the research was to identify how Brazilian public organizations realize the model of GC.The methodology used was an exploratory study through a literature search for jobs available in print and digital media in academic portals on the internet about GC in public organizations in Brazil. The results show that: there is the vast majority of organizations surveyed neither explicit nor formalization of GC. In addition, with respect to the strategic intent of the practices, the GC is still not a priority for most
} 
public organizations, which still focus their actions on tactical processes for solving everyday problems in obtaining activities, facilitation, learning, storage and dissemination of knowledge.

Key-words: knowledge management; exploratory study; brazilian public organizations.

\section{Referências}

ANGELONI, Maria T. e AUTORES. Gestão do Conhecimento no Brasil: casos, experiências e práticas de empresas públicas. Rio de Janeiro: Qualitymark, 2008.

BATISTA, Fábio F. Governo que aprende: gestão do conhecimento em organizações do executivo federal. Texto para discussão no 1022. Brasília: [s.n.], 2004.

; QUANDT, Carlos O.; PACHECO, Fernando F.; TERRA, José C. C. Gestão do conhecimento na administração pública. Texto para discussão no 1095. Brasília: [s.n.], 2005.

. O Desafio da Gestão do Conhecimento nas áreas de Administração e Planejamento da Instituições Federais de Ensino Superior (IFES). Texto para discussão no 1181. Brasília: [s.n.], 2006.

; XAVIER, Antonio C. da R.; MENDES, Luiz Carlos; ROSENBERG, Gerson. Gestão do Conhecimento em Organizações Públicas de Saúde. Texto para discussão no 1316. Brasília: [s.n.], 2007.

Modelo de Gestão do Conhecimento para a Administração Pública Brasileira: como implementar a gestão do conhecimento para produzir resultados em benefício do cidadão. Brasília: Ipea, 2012.

BOSE, R. Knowledge management-enabled health care management systems: capabilities, infrastructure, and decisionsupport. Expert Systems with Applications, v. 24, n. 1, p. 59-71, 2003. crossref

BOYATIZIS, R. The competent manager: A model of effective performance. New York: Wiley, 1982.

BRITO, Lydia M. P. Gestão de competências, gestão do conhecimento e organizações de aprendizagem: instrumentos de apropriação pelo capital do saber do trabalhador. Fortaleza: Imprensa Universitária, 2005.

; OLIVEIRA, Patrícia W. S. de; CASTRO, Ahiram B. C. de. Gestão do Conhecimento numa Instituição Pública de Assistência Técnica e Extensão Rural do Nordeste do Brasil. Revista de Administração Pública, vol. 46, nº 5, p.1341-1366. Rio de Janeiro: Fundação Getúlio Vargas, 2012.

CASTRO, Ahiram B. C de. Gestão do conhecimento: um estudo em uma instituição pública de assistência técnica e extensão rural. 2011. 150 f. Dissertação (Mestrado Profissional em Administração). Universidade Potiguar. Natal, 2011.

CAVAlCANTI, Marcos do C. B.; BALCEIRO, Raquel B.; GOMES, Elisabeth B. P. A prática da Gestão do Conhecimento em Empresas Públicas. Rio de Janeiro: e-papers, 2002.

COSTA, Ivani; VASCONCELOS, Ana C. F. de; CANDIDO, G. A. Diagnóstico de gestão do conhecimento como mecanismo para criação de valor: um estudo exploratório no SEBRAE-PB. Revista Gestão Industrial, Paraná, v. 5, n. 2: $\quad 2009 . \quad$ UTFPR, Disponível em: <http://www.pg.cefetpr.br/depog/periodicos/index.php/revistagi/article/viewArticle/346〉. Acessado em: 10 set. 2014.

COSTA, I. C. dos. Gestão do Conhecimento: um estudo comparativo entre organizações do Nordeste Brasileiro. 2014. 125 f. Dissertação (Mestrado Profissional em Administração). Universidade Potiguar. Natal, 2014.

COSTA, C. G. Aprendizagem no Trabalho: uma análise da percepção de gestores públicos. 2014. 113 f. Dissertação (Mestrado Profissional em Administração). Universidade Potiguar. Natal. 2014.

CHOO, Chun W. A Organização do conhecimento: como as organizações usam a informação para criar significado, construir conhecimento e tomar decisões. São Paulo: Senac, 2003.

DUTRA, Joel de Souza (Org.). Gestão por competências. São Paulo: Gente, 2002.

. Competências. São Paulo: Atlas, 2004. 
DRUCKER, Peter F. e outros. Aprendizado organizacional. São Paulo: Campus, 2000.

FLEURY, Maria Tereza. Aprendizagem e Inovação Organizacional. São Paulo: Atlas,1995.

FRIGOTTO, Gaudêncio. Educação e Crise do Capitalismo Real. São Paulo: Cortez, 1999. e outros. Neoliberalismo, Qualidade Total e Educação. Petrópolis/RJ: Vozes, 1995.

GALVÃO, Alcedo P. Gestão do conhecimento em empresa internacional de energia. 2012. 131 f. Dissertação (Mestrado Profissional em Administração). Universidade Potiguar. Natal, 2012.

Gil, A. C. Métodos e Técnicas de Pesquisa Social. $6^{\circ}$ ed. São Paulo: Atlas, 2008.

GOMES, Marcus V. D. A gestão do conhecimento na administração pública: paradigma para o Judiciário Fluminense. Trabalho de Conclusão de Curso de Pós-Graduação em Administração Judiciária. Fundação Getúlio Vargas - FGV. Rio de Janeiro, 2005.

GONZAGA, Aécio. Gestão do Conhecimento em Multinacional Brasileira de Energia. 2014. 127 f. Dissertação (Mestrado Profissional em Administração). Universidade Potiguar. Natal, 2014.

HAMEL, Gary e PRAHALAD, C.K. Competindo pelo Futuro: estratégias inovadoras para obter o controle do seu setor e criar mercados de amanhã. São Paulo: Campus, 1995.

LE BOTERF, G. Desenvolvendo a competência dos profissionais. Porto Alegre: Artmed Editora, 2003.

MARQUES, Fernanda. Gestão de Pessoas: fundamentos e tendências. Brasília: DDG/ENAP, 2015.

MELHOR REVISTA DE GESTÃo DE PESSOAS: especial 2010. São Paulo: Editora Segmento, 2010.

MCCLELLAND, D. Testing for Competence Rather Than for Intelligence. American Psychologist, January, 1973. crossref

OLIVEIRA, Djalma de P.R.de. Planejamento estratégico. São Paulo: Atlas, 2004.

OLIVEIRA, Josicleide A. Gestão do conhecimento: estudo de caso em um hospital universitário de ensino. 2011.185 f. Dissertação (Mestrado Profissional em Administração). Universidade Potiguar. Natal, 2011.

PERRENOUD, Philippe e outros. As competências para ensinar no século XXI: a formação dos professores e o desafio da avaliação. Porto Alegre: Artmed, 2002.

POLANCZYK, C. A. Protocolos assistenciais como estratégia de adesão às melhores práticas clínicas e otimização de recursos. Concurso Inovação na Gestão Pública Federal. Brasília: Escola Nacional de Administração Pública - Enap. Disponível em: <http://repositorio.enap.gov.br/bitstream/handle/1/342/067_02.pdf?sequence=1\&isAllowed=y > Acessado em: 24 jan. 2015.

QUINN, Robert E. Competências gerenciais. Rio de Janeiro: Elsevier, 2003.

RAMOS, Marise Nogueira. A Pedagogia das Competências: Autonomia ou Adaptação? São Paulo: Cortez, 2001.

RUAS, Roberto, ANTONELlO, Claudia Simone e BOFF, Luiz Henrique. Aprendizagem Organizacional e Competências. Porto Alegre: Bookman, 2005.

RUNTE, Glória I. B. da C. Gestão do Conhecimento os desafios da implantação de um modelo integrado o caso ANS. Dissertação de Mestrado em Administração Pública. Fundação Getúlio Vargas - FGV. Rio de Janeiro, 2011.

SCHLESINGER, Cristina C. B. et al. Gestão do conhecimento na administração pública. [S.1.]: Instituto Municipal de Administração Pública - IMAP, 2008.

SENGE, Peter R. A 5a Disciplina: caderno de campo. Rio de Janeiro: Qualitymark, 1995.

STRAUHS, Faimara do R. et al. Gestão do conhecimento nas organizações. Curitiba: Aymará Educação, 2012.

TAVARES, W. R. Gestão do conhecimento: Educação e Sociedade do Conhecimento. São Paulo: Ícone, 2010. 
TERRA, José C. C. Gestão do conhecimento: o grande desafio empresarial. Rio de Janeiro: Elsevier, 2005.

TRIGUEIRO, Francisco M. C.; MARQUES, Neiva de A. Teoria da Administração I. $2^{\circ}$ ed. reimp. Florianópolis: Departamento de Ciências da Administração, UFSC, 2012.

ZARIFIAN, Philippe. Objetivo Competência: por uma nova lógica. São Paulo: Atlas, 2001.

\section{Dados dos autores:}

Nome completo: Ahiram Brunni Cartaxo de Castro

Filiação institucional: Instituto Federal de Educação, Ciência e Tecnologia do Rio Grande do Norte - IFRN, RN

Departamento: Diretoria de Administração

Função ou cargo ocupado: Administrador

Endereço completo para correspondência: Rua Otávio Gomes de Castro, 69, Centro,

Parnamirim/RN. Cep. 59140-140

Telefones para contato: (84) 99172.8680

e-mail: brunnicastro@hotmail.com

Nome completo: Lydia Maria Pinto Brito

Filiação institucional: Universidade Potiguar - UNP, RN

Departamento: Programa de Pós-graduação em Administração Stricto Sensu - Doutorado e

Mestrado

Função ou cargo ocupado: Professora Titular

Endereço completo para correspondência: Rua Pereira Valente, 720, apto. 1000. Meireles/CE. Cep. 60.160-250.

Telefones para contato: (85)99145.2596

e-mail: lydiampbrito@yahoo.com.br

Submetido em: 21-11-2015

Aceito em: 15-06-2016 